\title{
Behavior Analysis of Water Droplets in Wake Flow of a Transonic Cascade
}

\author{
Lanxin Sun 1,3 , Tao Sun², Yigang Luan² \\ ${ }^{1}$ College of Nuclear Science and Technology, Harbin Engineering University, Harbin 150001, China \\ ${ }^{2}$ College of Power and Energy Engineering, Harbin Engineering University, Harbin 150001, China \\ ${ }^{3}$ Fundamental Science on Nuclear Safety and Simulation Technology Laboratory, Harbin 150001, \\ China \\ E-mail: sunlanxin@126.com
}

Key Words: Two-phase flow; Water injection; Droplet breakup; Wake flow; Aerodynamic breakup.

\begin{abstract}
The compression process in an axial compressor with water injection is a complex two-phase flow involving many changes of water existing forms. Boundary conditions close to the real ones of water droplets on compressor blade are extremely important for numerical simulation. Water droplet impinging and stick to surfaces of compressor rotors or stators will form into water film, and then shed off from the trailing edge of the blade. For a rotor with high rotating speed and a stationary stator, the shedding water film from the trailing edge may experience different aerodynamic breakups study of which is very import for the application of water injection technique to compressor. In this paper, comparison and analysis on breaking up of big droplets in the wakes of rotor and stator are carried out through designing appropriate numerical experiments. In order to obtain the comparable wake flows for rotor and stator separately which are elementary for this study, a transonic rotor, NASA rotor 37, was chosen for the present study to produce nearly uniform wake flows of rotor and stator. Research found that movement and breakup of big droplets in wake flows is entirely different for rotor and stator, and this discovery is of great significance.
\end{abstract}

\section{Introduction}

Water injection technique has shown to improve gas turbine performance and, has been successfully employed on industrial gas turbines in recent years [1-2]. Many studies on this technique show clearly advantages of water injection into compressor and also identify the related parameters influencing performance of compressor and overall gas turbine [3-7]. Recently much work has been done to explain flow behavior in multistage compressors using simplified models such as mean line and stage-stacking [8-10]. Numerical analysis on film formation on compressor blade shows that the ingested water impacted on the blade pressure side, and only little droplet deposition was found on the suction side [11]. Some of the unknown effect relating to droplet dynamics within the rotor blade passage in Payne and White study was investigated analytically [12].

When impinging onto blade surfaces of compressor, water droplets will stick to them and then form into water film. The film will shed off from the trailing edge of the blade due to the moving inertia as well as the aerodynamic shear force. In the present study, based on the CFD method, three-dimensional flow simulation is carried out for an isolated transonic compressor rotor NASA rotor 37 to study the problem of water film shedding from blade trailing. Structural meshing method is used to discretize the computational domain, and CAB breakup model is adopted to consider the aerodynamic breakup. Appropriate numerical experiment is designed first to obtain the comparable wake flow for rotor and stator separately. And then the varying behavior of the shedding water film is studied and analyzed to see whether it depends on the blade being rotating or stationary. 


\section{Numerical Method and Physical Model}

\subsection{Governing Equations and Turbuence Model}

Two-way coupling between the two phases of gas phase and droplet phase should be considered to properly solve the interaction process of two-phase flow. The Eulerian-Lagrangian model is chosen to capture the trajectory of moving droplet, by Eulerian method to solve the equations of air and water vapor mixture, and Lagrangian method the equations of water droplet discrete phase.

1) Governing equations of the continuous phase

Governing equations for mass, momentum and energy of the continuous phase are written as

$$
\begin{gathered}
\frac{\partial \rho}{\partial t}+\nabla \cdot(\rho \vec{u})=S_{\mathrm{m}} \\
\frac{\partial}{\partial t}(\rho \vec{u})+\nabla \cdot(\rho \vec{u} \vec{u})=-\nabla p+\nabla \cdot(\overline{\bar{\tau}})+\vec{F} \\
\frac{\partial}{\partial t}\left(\rho h_{\mathrm{t}}\right)+\nabla \cdot\left(\vec{u}\left(\rho h_{\mathrm{t}}+p\right)\right)=\nabla \cdot(\lambda \nabla T+(\overline{\bar{\tau}} \cdot \vec{u}))+\vec{u} \cdot \vec{F}+S_{\mathrm{h}}
\end{gathered}
$$

2) Governing equations for the dispersed phase

Forces acting on droplet particles can examine only the aerodynamic drag force, the centrifugal force and the Coriolis force, and ignore other much smaller ones. Then the equation of droplet motion can be written as:

$$
m_{\mathrm{p}} \frac{\mathrm{d} \vec{u}_{\mathrm{p}}}{\mathrm{d} t}=\vec{F}_{\mathrm{D}}+\vec{F}_{\mathrm{R}}
$$

Aerodynamic shear breakup of big droplets is considered by CAB (Cascade Atomization and Breakup model) model. This model is development of the TAB model (Taylor Analogy Breakup model) [9] and is generally adopted for its robust feature in solveing secondary breakup of droplet. In the model, the droplet viscosity is assumed to act as a damping force and the surface tension a restoring force. The equation of deformation motion can be written as:

$$
\ddot{y}=\frac{5 \mu_{\mathrm{p}}}{\rho_{\mathrm{p}} r^{2}} \dot{y}+\frac{8 \sigma}{\rho_{\mathrm{p}} r^{3}} y+\frac{2 \rho_{\mathrm{g}} V_{\text {slip }}^{2}}{3 \rho_{\mathrm{p}} r^{2}}
$$

3) Turbulence model

The standard $k-\varepsilon$ turbulence model is a semi-empirical model, with good robustness and small amount of calculation. It employs the turbulent kinetic energy equation ( $k$ equation) and turbulent kinetic energy dissipation rate equation ( $\varepsilon$ equation) to close the turbulent flow governing equations.

$$
\begin{aligned}
& \frac{\partial(\rho k)}{\partial t}+\nabla \cdot(\rho \vec{u} k)=\nabla \cdot\left[\left(\mu+\frac{\mu_{\mathrm{t}}}{\sigma_{\mathrm{k}}}\right) \nabla k\right]+P_{\mathrm{k}}-\rho \varepsilon \\
& \frac{\partial(\rho \varepsilon)}{\partial t}+\nabla \cdot(\rho \vec{u} \varepsilon)=\nabla \cdot\left[\left(\mu+\frac{\mu_{\mathrm{t}}}{\sigma_{\varepsilon}}\right) \nabla \varepsilon\right]+\frac{\varepsilon}{k}\left(C_{\varepsilon 1} P_{\mathrm{K}}-C_{\varepsilon 2} \rho \varepsilon\right)
\end{aligned}
$$

Scalable wall function is selected to solve the flow near the wall, and this method can be applied to meshes with arbitrary precision.

\section{Physical Model and Mesh Topology}

A transonic rotor NASA rotor 37 [13] was chosen for this investigation. The compressor contains 36 blades, the design rotating speed, mass flow rate, total pressure ratio are $17188.7 \mathrm{rpm}, 20.19 \mathrm{~kg} / \mathrm{s}$, 2.106 respectively. Multi-block structured grid topology was used and a grid containing 0.62 million elements was applied to discretize the cascade passage domain (Figure 1a). High-quality $\mathrm{H}$ grid was utilized for inlet and outlet blocks, while good J grid was utilized for mid-block with $20 \mathrm{O}$ grid layers around the blade (Figure 1b). The tip-clearance was in consideration with enough grid elements in span wise direction (Figure 1c). 


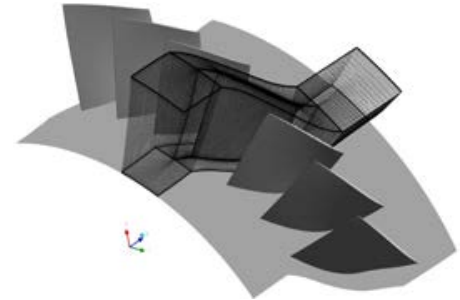

(a) Domain grid

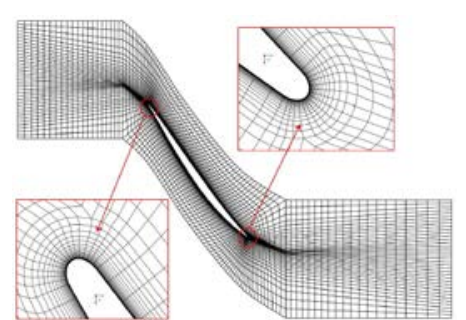

(b) B2B grid

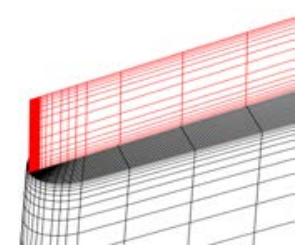

(c) tip clearance grid

Fig. 1 Computational grids for NASA Rotor 37

\section{Numerical Simulations and Results}

The calculation condition is at the design point. Total pressure and total temperature were given at the compressor inlet with the value of test data. Non-slip and adiabatic conditions were imposed on walls such as hub, shroud and blade. At the exit boundary, condition of static pressure was given. Large droplets are released from the upper half of the trailing edge of the blade with an initial droplet diameter of $45 \mu \mathrm{m}$, at the initial velocity $30 \mathrm{~m} / \mathrm{s}$ approximately along the tangential direction of the blade.

Moving droplets impinging onto the blade surfaces of rotor or stator will form into water film which will next shed off from the trailing edge under the gas blowing and then breakup into varying secondary droplets in the wake flow. Because of the high rotating speed blade, breakup behavior of shedding film from a rotor will experience a very different situation from that of a stator. In order to be able to compare and analyze the breakup situation of big droplets in wake flows between rotors and stators, numerical experiment is designed specially to produce approximately consistent wake flows which own the same relative velocity distribution in the wake flow field. In order to ensure the flow field parameters (mainly the relative velocity) of wake flows for both rotors and stators are similar, mass flowrate condition is imposed on to the stator inlet with the same value as that of the rotor. Solutions of inlet relative velocity and total temperature distribution of the rotor are assigned to the stator inlet, and the outlet static pressure of the rotor is assigned to the stator outlet.

Figure 2 shows the relative Mach number distributions on B2B sections of 75\% span for rotor and stator separately, from which it can be seen that parameter distributions after the trailing edge of rotor and stator are very similar and can be used to compare and analyze droplet motion and breakup in the wake flow.

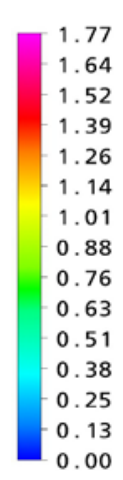

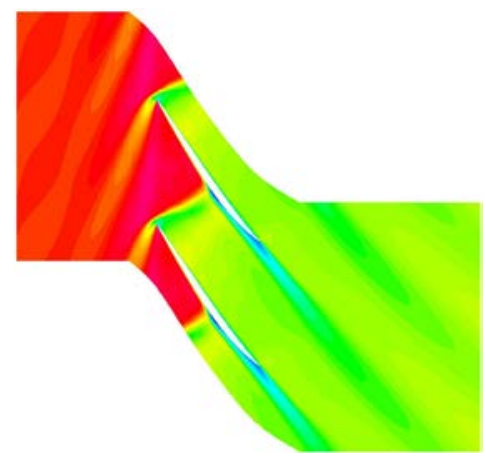

(a) rotating blade

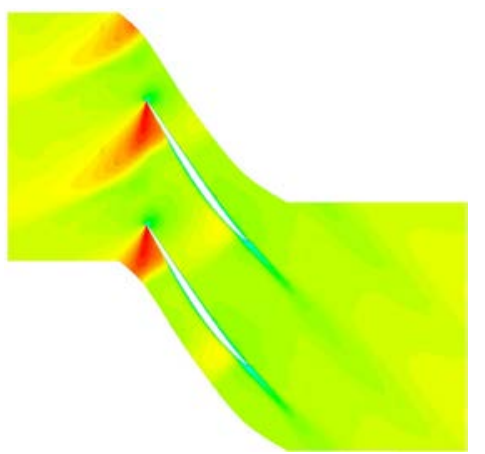

(b) non-rotating blade

Fig. 2 Similar Mach number distribution downstream of the rotating/non-rotating blades

Figure 3 shows the trajectory and breaking up of big droplets shedding from trailing edges of rotor and stator, from which it can be seen that the droplets behaviors for both blades are of very different results. Big droplets shedding from the rotor trailing edge encounter violent breaking up under the effect of aerodynamic force, and almost all the breakup of big droplets appear near the aft trailing edge. It produces a size distribution less than $20 \mu \mathrm{m}$, most of the droplet diameter is less than $15 \mu \mathrm{m}$, and the droplet trajectory deviates from the airflow wake and appears a relative lag.

From Figure 3, it can also be seen that big droplets shedding from the stator trailing edge encounter the same violent breaking up under the effect of aerodynamic force, and almost all the breakup of big droplets appear near the aft trailing edge. But it produces a size distribution about 
$30 \mu \mathrm{m}$, and the droplet trajectory shows no deviation from the airflow wake and no such a relative lag like that of the rotor. By comparison to those big droplets, Figure 4 shows the droplet trajectory of $2 \mu \mathrm{m}$ in diameter released from the rotor trailing edge, which indicates that very small droplets can follow the airflow relatively well and their trajectory is consistent with the vortex behind the trailing edge.

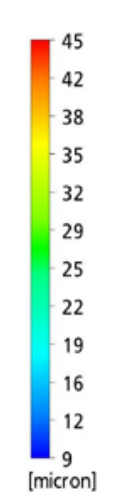

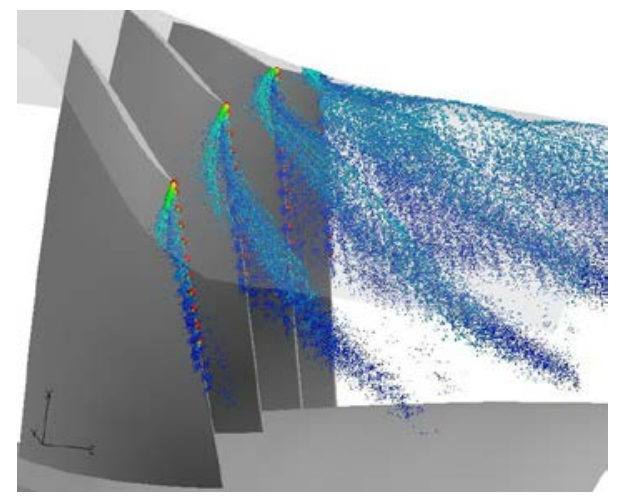

(a) rotating blade

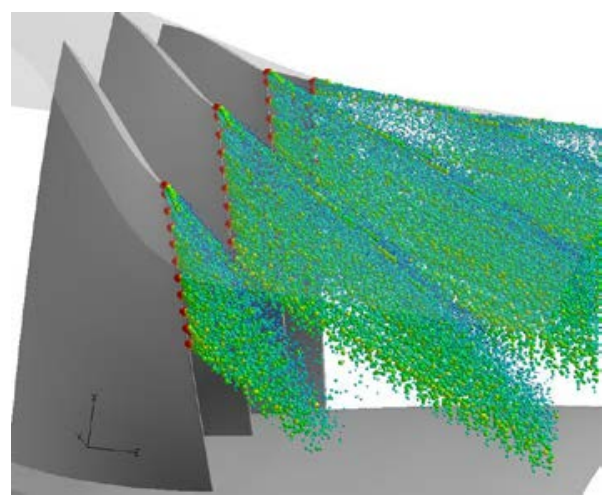

(b) non-rotating blade

Fig. 3 Changes of trajectories and sizes of released big droplets from trailing edge of the rotating and non-rotating blades

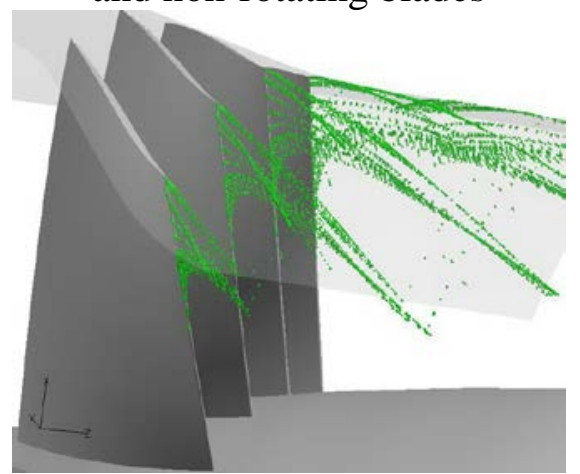

Fig. 4 Trajectory small droplets in wake flow of the rotating blade

With the help of velocity triangles of air and droplet (shown in Figure 5), comparison and analysis of motion and breakup of large droplets in the wake flow of rotor and stator are carried out. The moment water film or water droplets shed off from the rotor trailing edge, its circumferential velocity $U_{\mathrm{Pc} 1}=\omega r$ is the same with that of the air flow $U_{\mathrm{c}}$, its relative velocity $U_{\mathrm{Pw} 1}$ is far smaller than that of the air flow $U_{\mathrm{w}}$, and its absolute velocity $U_{\mathrm{Pa} 1}$ is much different with that of the air flow $U_{\mathrm{a}}$.

After water film or large droplet shedding off from the trailing edge of rotor, due to the aerodynamic force its circumferential velocity decreases rapidly, and its relative velocity increases rapidly. Then violent breakup occurs, but in a very short period of time the directions of relative velocity and absolute velocity of water droplets get close to that of airflow, and the velocity slip between the two phases are not large enough for the droplets to deform seriously and breakup again.

For water film or large droplet shedding off from the trailing edge of stator, violent breakup also occurs during the aerodynamic acceleration process, but there is no such a large transverse shear force as that of the rotor because of the lag resulted from rotating circumferential velocity. The diameter of newly produced droplets is relatively large compared to that of the rotor, and their moving trajectory is roughly the same as the wake flow with no clear offset. 

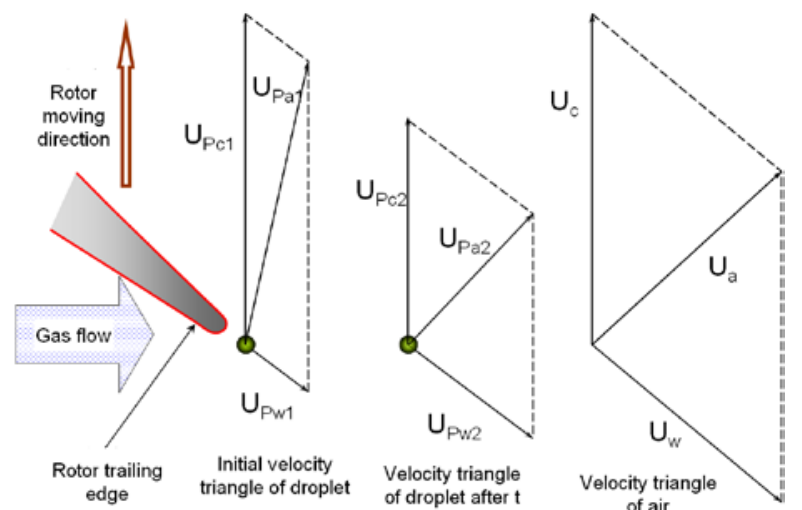

Fig. 5 Change of velocity triangle for the released droplets of the rotating blade

\section{Conclusions}

Study of water content shedding off from blade trailing edges is very import for the technique of water injection into compressor. Comparison and analysis on different behavior of big droplets in the wakes of rotor and stator are studied by numerical experiments. The conclusions are as follows:

1) For the rotor, big shedding droplets from the trailing edge encounter violent breaking up, almost all the breakup appears behind trailing edge and produces a size distribution of less than $20 \mu \mathrm{m}$, and the droplet trajectory deviates from the airflow wake. Very small droplets can follow the airflow well and their trajectory is consistent with the vortex streamline behind the trailing edge.

2) For the stator, breakup of big shedding droplets is similar to that of the rotor, but it produces a size distribution about $30 \mu \mathrm{m}$, and the droplet trajectory shows no deviation from the airflow wake.

3) After water film or large droplet shedding off from the trailing edge of rotor, its circumferential velocity decreases rapidly, but its relative velocity increases quickly, and this results violent breakup. Phenomenon is the same for the stator, but the diameter of new droplets is larger compared to the rotor, and trajectory is in line with the wake flow with no clear offset.

\section{Acknowledgement}

The authors would like to acknowledge the support of National Natural Science Foundation of China, Grant No. 51409067; and Harbin Engineering University Foundation HEUFN1306. Authors would also like to thank reviewers for their comments which help us improve the quality of this paper.

\section{References}

[1] Jolly, S. and Cloyd, S., 2003, "Performance Enhancement of GT24 with Wet Compression," PowerGen International, December 9-11, Las Vegas, NV, USA

[2] Bhargava, R. K., Meher-Homji, C. B., Chaker, M. A., Bianchi, M., Melino, F., Peretto, A., and Ingistov, S., 2005, "Gas Turbine Fogging Technology - A State of the Art Review, Part II: Overspray Fogging - Analytical and Experimental Aspects”, ASME Paper No. GT2005-68337

[3] Bhargava, R. and Meher-Homji, C. B., 2002, "Parametric Analysis of Existing Gas Turbines with Inlet Evaporative and Overspray Fogging”, ASME Paper No. GT2002-30560

[4] Zheng, Q., Sun, Y., Li, S., and Wang, Y., 2002, "Thermodynamic Analyses on Wet Compression Process in the Compressor of Gas Turbine”, ASME Paper No. GT2002-30590

[5] Zheng, Q., Li, M., and Sun, Y., 2003, “Thermodynamic Performance of Wet Compression and Regenerative (WCR) Gas Turbine”, ASME Paper No. GT2003-38517

[6] Sexton, W. R. and Sexton, M. R., 2003, "The Effects of Wet Compression on Gas Turbine Engine Operating Performance”, ASME Paper No. GT2003-38045

[7] Shao, Y. and Zheng, Q., 2005, “The Entropy and Exergy Analyses of Wet Compression Gas Turbine”, ASME Paper GT2005-68649

[8] Bhargava, R. K., Bianchi, M., Melino, F., Peretto, A., and Spina, P. R., 2008, "Influence of 
Compressor Performance Maps Shape on Wet Compression”, ASME Paper No. GT2008-50761

[9] Bhargava, R. K., Bianchi, M., Chaker, M., Melino, F., Peretto, A., and Spina, P. R., 2009, “Gas Turbine Compressor Performance Characteristics During Wet Compression - Influence of Polydisperse Spray”, ASME Paper No. GT2009-59907

[10] Khan, J. R. and Wang, T., 2009, “Overspray Fog Cooling in Compressor Using Stage-Stacking Scheme with Non-Equilibrium Heat Transfer Model for Droplet Evaporation,” ASME Paper No. GT2009-59590

[11] Nikolaidis, T., Pilidis, P., Teixeira, J. A., and Pachidis, V., 2008, "Water film formation on an axial Flow Compressor Rotor Blade,” ASME Paper No. GT2008-50137

[12] Williams, J. and Young, J. B., 2006, "Movement of Deposited Water on Turbomachinery Rotor Blade Surfaces”, ASME Paper No. GT2006-90792

[13] Reid, L. and Moore, R. D., 1978, "Design and Overall Performance of Four Highly-Loaded, High-Speed Inlet Stages for an Advanced, High-Pressure-Ratio Core Compressor”, NASA TP-1337 